\title{
The power spectrum of the circular noise
}

\author{
Daniel Müller \\ Instituto de Física, UnB \\ Cxp 04455, 70919-970, Brasília DF \\ Brazi
}

\begin{abstract}
The circular noise is important in connection to Mach's principle, and also as a possible probe of the Unruh effect. In this letter the power spectrum of the detector following the Trocheries-Takeno motion in the Minkowski vacuum is analytically obtained in the form of an infinite series. A mean distribution function and corresponding energy density are obtained for this particular detected noise. The analogous of a non constant temperature distribution is obtained. And in the end, a brief discussion about the equilibrium configuration is given.
\end{abstract}

KEY WORDS: circular Unruh effect

The non inertial vacuum, and the noise associated to it, has been extensively studied. It is called Fulling-DaviesUnruh effect, after the discoverers [1].

The more simple situation of proper constant acceleration, is already very well understood. For instance, a free scalar field can be quantized in Rindler coordinates. The vacuum obtained in these coordinates is unitary inequivalent to the Minkowski vacuum.

The monopole type detector introduced by DeWitt [2], can give a meaning to these vacua. A detector at rest in Rindler coordinates is equivalent to an accelerated one in Minkowski space.

As a consequence of Poincaré invariance, a detector in inertial motion in vacuum, does not get excited. This can also be understood in the detailed balance context of [3]. The authors used the DDC [4] formalism to obtain the Einstein coefficients for spontaneous excitation and emition. For non inertial motion the balance of the coefficients is upset, resulting in the Unruh effect. The thermal character of the noise in the KMS sense, and the verification of other consistencies are reviewed in [5]. In [6] it is shown the connection between the absorptions of particles and the noise to which the detector is submitted.

The rotational motion is different. Besides its own importance connected to the concept of inertia, this motion is of experimental relevance, because it provides a possible verification of the Unruh effect [7]. The DDC formalism was also used in the context of rotation to obtain the spontaneous excitation of the detector, the circular Unruh effect [8]. In the very interesting work [9], De Lorenci and Svaiter develop the quantization in Trocheries-Takeno [10 coordinates, resulting in a non trivial vacuum. Davies et al also obtain a very interesting result 11] concerning rotational motion. Since now, the De Lorenci-Svaiter-Trocheries-Takeno vacuum is the only one available for rotation. I do not attempt to enter into the vacuum question.

In this letter, an analytical expression for the power spectrum in the form of an infinite series is obtained, a result which has not yet appeared elsewhere. In [9] the transition rate obtained here, is left over as an integral. I suppose a detector in Minkowski vacuum, constrained to a Trocheries-Takeno type motion. It should be stressed that by following the procedure outlined in this letter, inside the light cylinder of [11], results in a transition rate similar to equation (7) below.

It is assumed a monopole type coupling of the detector and field,

$$
L_{I}=-H_{I}=M \phi(x(\tau)),
$$

where $M$ has energy levels. We require both the detector and the field to obey Schrödinger's unitary time evolution. Let us assume that in the very early past the detector is in the ground state with energy $E_{0},\left|E_{0}\right\rangle$ and that the field is in the (Minkowski) vacuum state $\left|0_{M}\right\rangle$. As the detector moves, field and detector will undergo transitions to various states, in a trajectory dependent fashion [12]. Since one does not care about the field's final state, the unknown final state of the field should be traced out from the transition probability for thetransitions in question. This process

*Electronic address: muller@fis.unb.br 
yields, in first order perturbation theory the following expression for the transition rate

$$
\begin{aligned}
& G^{+}\left(x_{1}, x_{2}\right)=\frac{1}{4 \pi^{2}}\left(\frac{1}{-\left(t_{1}-t_{2}\right)+\left(\overrightarrow{x_{1}}-\overrightarrow{x_{2}}\right)^{2}}\right) \\
& G^{+}\left(x\left(s_{1}\right), x\left(s_{2}\right)\right)=\left\langle 0_{M}\left|\phi^{\dagger}\left(x^{\mu}\left(s_{1}\right)\right) \phi\left(x^{\mu}\left(s_{2}\right)\right)\right| 0_{M}\right\rangle \\
& P(E)=C(E) \lim _{\tau_{0} \rightarrow \infty} \frac{1}{2 \tau_{0}} \int_{-\tau_{0}}^{+\tau_{0}} \int_{-\tau_{0}}^{+\tau_{0}} e^{-i E\left(s_{1}-s_{2}\right)} G^{+}\left(x\left(s_{1}\right), x\left(s_{2}\right)\right) d s_{1} d s_{2},
\end{aligned}
$$

where $E>0$ is the energy difference between the initial and final states of the detector and $G^{+}\left(x, x^{\prime}\right)$ is the positive frequency Wightman function.

$C(E)$ depends on the internal constituency of the detector and a detailed discussion of it is given in [3] and [8]. While the second term in (2) corresponds to the noise this detector is submitted to, that's to say, on the way the field fluctuates as seen by the observer in his trajectory $x^{\mu}(\tau)$.

It is an easy exercise to show that a detector at rest in the Trocheries-Takeno coordinates $\left(d r^{\prime}=0, d \theta^{\prime}=0, d z^{\prime}=0\right)$

$$
\begin{aligned}
& t=t^{\prime} \cosh \Omega r^{\prime}-r^{\prime} \theta^{\prime} \sinh \Omega r^{\prime} \\
& r=r^{\prime} \\
& \theta=\theta^{\prime} \cosh \Omega r^{\prime}-\frac{t^{\prime}}{r^{\prime}} \sinh \Omega r^{\prime} \\
& z=z^{\prime}
\end{aligned}
$$

according to (2), has a transition rate given by

$$
P(E)=-\frac{1}{16 \pi^{2} \cosh ^{2} \Omega r} \int_{-\infty}^{+\infty} \frac{e^{-i E s}}{(s / 2)^{2}-[r / \cosh (\Omega r)]^{2} \sin \left[(s / 2) \sinh (\Omega r) r^{-1}\right]^{2}} d s,
$$

where $s=t_{2}^{\prime}-t_{1}^{\prime}$ is the proper time between two points in the trajectory of the detector. After a change in the integration variable the above transition rate, is written as

$$
P(E)=-\frac{\omega}{8 \pi^{2} \gamma} \int_{-\infty}^{\infty} \frac{\exp [-i(2 E /(\gamma \omega)) x] d x}{(x)^{2}-v^{2} \sin (x)^{2}}
$$

where

$$
\begin{aligned}
& \omega=\frac{\tanh (\Omega r)}{r}, \omega \simeq \Omega, r \rightarrow 0 \\
& v=\omega r, v<1 \\
& \gamma=\cosh (\Omega r)=\frac{1}{\sqrt{1-v^{2}}} .
\end{aligned}
$$

The fact that $v<1$, comes from the type of trajectory that the detector is following. In this sense, this trajectory is more physical, because the detector is not allowed to travel faster than the speed of light. In (2), the $1 /\left(2 \tau_{0}\right)$ factor is associated to the adiabatic switching of the detector and the usual $-i \epsilon$ prescription is related to its size. As $v<1$, and the integral is over the real axis, the integrand in (5) can be written as

$$
P(E)=-\frac{\omega}{8 \pi^{2} \gamma} \int_{-\infty}^{+\infty} d x \frac{\exp [-i 2(E /(\gamma \omega)) x]}{(x-i \epsilon)^{2}} \sum_{n=0}^{\infty}\left(\frac{v \sin (x-i \epsilon)}{x-i \epsilon}\right)^{2 n} .
$$

After a binomial expansion, this last integral is evaluated using ordinary residue calculus

$$
P(E)=\frac{\omega}{2 \pi \gamma v} \sum_{n=1}^{\infty} \sum_{k=1}^{\infty} \frac{[v(-E /(\gamma \omega)+k)]^{2 n+2 k-1} \Theta(-E+k \omega \gamma)}{(-1)^{n-1}(2 n+2 k-1)(n-1) !(n+2 k-1) !},
$$

where $\Theta(x)$ is the Heaviside step function, which indicates that the rotational motion is the thermal reservoir. The time scales of the detector $E$, and the (proper) period of rotation of the detector $\gamma T^{\prime}=2 \pi / \omega$ are singled out in (7), a property also shown in [9] and [11]. 
As a spectrum, (7), can be divided by the energy $E$ and an effective distribution function for the scalar particles can be obtained

$$
n(E)=\frac{\omega}{2 \pi \gamma v E} \sum_{n=1}^{\infty} \sum_{k=1}^{\infty} \frac{[v(-E /(\gamma \omega)+k)]^{2 n+2 k-1} \Theta(-E+k \omega \gamma)}{(-1)^{n-1}(2 n+2 k-1)(n-1) !(n+2 k-1) !} .
$$

Effective here is in a certain sense, that the distribution function is dependent on the measuring apparatus. Other consequences connected to the motion, should be perceived by other types of detectors. Only the detectable properties are taken into account. This distribution function is understood as the mean number of particles per volume, with energy $E$ perceived by the detector. In the following, density will be assumed to be per unit volume.

In this spirit, the total energy density of the scalar particles is given by the following integral, which can be written in terms of the hypergeometric function

$$
\begin{aligned}
& E_{t}=\int_{0}^{\infty} E n(E) d E=\frac{\omega}{2 \pi \gamma v} \sum_{n=1}^{\infty} \sum_{k=1}^{\infty} \int_{0}^{k \omega \gamma} d E \frac{[v(-E /(\gamma \omega)+k)]^{2 n+2 k-1}}{(-1)^{n-1}(2 n+2 k-1)(n-1) !(n+2 k-1) !} \\
& E_{t}=\frac{\omega^{2}}{2 \pi v} \sum_{n=1}^{\infty} \sum_{k=1}^{\infty} \int_{0}^{k} d x \frac{[v(-x+k)]^{2 n+2 k-1}}{(-1)^{n-1}(2 n+2 k-1)(n-1) !(n+2 k-1) !} \\
& E_{t}=\frac{1}{2 \pi r^{2}} \sum_{n=1}^{\infty} \sum_{k=1}^{\infty} \frac{(v k)^{2(n+k)}}{(-1)^{n-1}(2 n+2 k)(2 n+2 k-1)(n-1) !(n+2 k-1) !} \\
& E_{t}=\frac{1}{2 \pi r^{2}} \sum_{k=1}^{\infty} \frac{(\tanh (\Omega r) k)^{2 k+2}{ }_{2} F_{3}\left(k+1 / 2, k+1 ; k+3 / 2, k+2,2 k+1 ;-\tanh (\Omega r)^{2} k^{2}\right)}{(k+1 / 2)(k+1)(2 k) !}
\end{aligned}
$$

where $\Omega$ plays a role similar to the temperature, and there is an explicit dependence on the coordinate $r$. This corresponds to a non constant temperature distribution. For instance, in the above expression (10), at the origin when $r=0$, the energy density is $E_{t}=0$, which makes sense, because the motion of detector when $r=0$ is inertial.

Next, the convergence of the infinite summation (9), is discussed. The second derivative with respect to $v$ of (9) is of the type

$$
\begin{aligned}
& \sum_{k=0}^{\infty}(v k)^{2 k+2} \sum_{n=0}^{\infty} \frac{\left(-v^{2} k^{2}\right)^{n}}{v^{2} \Gamma(n+2 k+1) n !}= \\
& =\sum_{k=0}^{\infty}(v k)^{2}(v k)^{2 k} \frac{J_{2 k}(2 k v)}{v^{2}(k v)^{2 k}}= \\
& =\sum_{k=1}^{\infty} k^{2} J_{2 k}(2 k v) \\
& \rightarrow \sum_{k} \frac{k^{3 / 2}}{\sqrt{4 \pi \sqrt{1-v^{2}}}}\left(\frac{v e^{\sqrt{1-v^{2}}}}{1+\sqrt{1-v^{2}}}\right)^{2 k},
\end{aligned}
$$

the last expression is valid asymptotically when $k \rightarrow \infty$ and $0<v<1$ [13]. In this asymptotic spirit, the summation (11) can be replaced by the integral over $k$, from 0 to $\infty$, which results in

$$
g(v)=\frac{3 \sqrt{\pi}}{4}\left|\ln \left(\frac{v e^{\sqrt{1-v^{2}}}}{1+\sqrt{1-v^{2}}}\right)\right|^{-5 / 2} .
$$

The original summation (9) is obtained by integrating the last equation two times in $v$

$$
\int_{0}^{v} d v^{\prime} \int_{0}^{v^{\prime}} d v^{\prime \prime} g\left(v^{\prime \prime}\right)
$$

which is plotted numerically in the following 


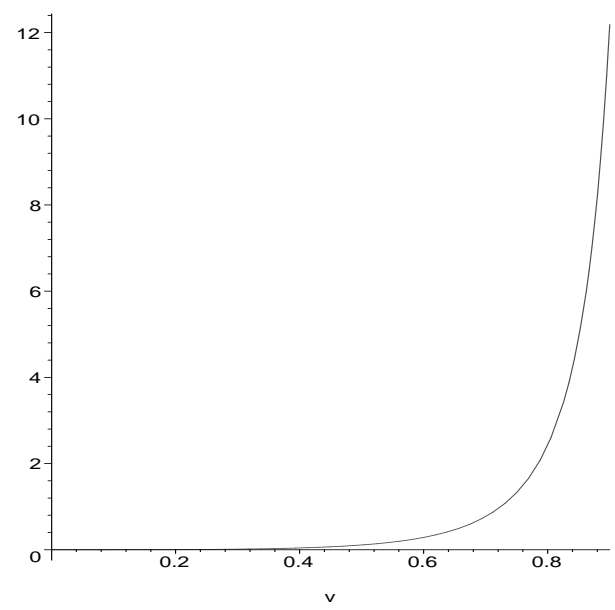

The above graphic indicates, in a indirect way, that the summation given in (9) and (10) converges, except when $v \rightarrow 1$. This divergence of the total energy density of the scalar particles perceived by the detector, is expected when the detector is moving (almost) with the velocity of light, $v \rightarrow 1$.

By summing the first few terms in (10), the following total energy density of scalar particles is obtained at a given $r$, for $\Omega=0.2$ and $\Omega=0.16$

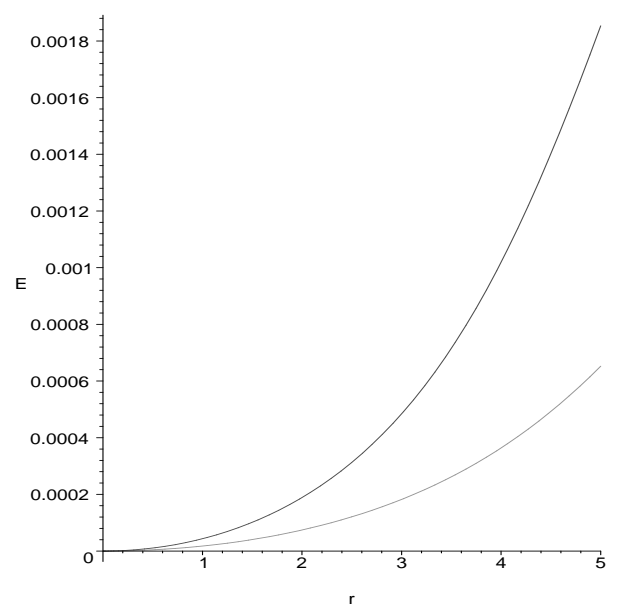

showing that for a same distance $r$ from the origin, there is an increase in the energy for larger values of $\Omega$.

It should be stressed that in spite that (10) describes a non constant temperature distribution, it is an equilibrium configuration. This interesting equilibrium concept is well known and is expected if the space being considered is not homogeneous and isotropic as in Trocheries-Takeno case, see for example 14]. The external condition that originates this motion is responsible for this apparent non equilibrium. The above construction avoids canonical quantization, and it could be usefull to a further understanding of external conditions.

\section{Ackowledgments}

The author acknowledges the brazilian agencies Funpe and Finatec for partial support, and an anonymous referee for improvements.

Note added in proof: It is a pleasure to thank Dr. Marcelo Schiffer which was my Msc. advisor.

[1] Unruh W G 1976 Phys. Rev. D 14 870; Davies P C W 1975 J. Phys. A 8 609; Fulling S A 1973 Phys. Rev. D 10 2850

[2] DeWitt B S 1979 in General Relativity, ed. S. W. Hawking and W. Israel (Cambridge University Press) p. 680 
[3] Audretsch J and Müller R 1994 Phys. Rev. A 501755

[4] Dalibard J, Dupont-Roc J. and Cohen-Tannoudji C. 1982 J. Phys. (Paris) 431617

[5] Takagi S 1988 Prog. Theor. Phys. 881

[6] Unruh W G and Wald R M 1984 Phys. Rev. D 291047

[7] Bell J S and Leinaas J M 1983 Nucl. Phys. B212 131; Leinaas J M 2001 Preprint hep-th/0101054

[8] Audretsch J, Müller J R and Holzmann M 1995 Class. Quant. Grav. 122927

[9] De Lorenci V A and Svaiter N F 1999 Found. Phys. 29 1233; De Lorenci V A, De Paola R D M and Svaiter N F 2000 Class. Quant. Grav. 174241

[10] Trocheries M G 1949 Phyl. Mag. 40 1143; Takeno H 1952 Prog. Theor. Phys. 7367

[11] Davies P C W, Dray T and Manogue C A 1996 Phys. Rev. D 534382

[12] Sciama D W, Candelas P and Deutsch D 1981 Adv. Phys. 30327

[13] Whittaker E T and Watson G N 1963 A Course of Modern Analysis, Cambridge at the University Press, p. 369.

[14] Stefani H 1993 General Relativity: An introduction to the theory of the gravitational field, Cambridge University Press, P. 80-83. 